Research Article

\title{
Existence of Multiple Periodic Solutions for Cubic Nonautonomous Differential Equation
}

\author{
Saima Akram (D), ${ }^{1}$ Allah Nawaz, ${ }^{1}$ Humaira Kalsoom $\left(\mathbb{D},{ }^{2}\right.$ Muhammad Idrees, ${ }^{3}$ \\ and Yu-Ming Chu iD ${ }^{4,5}$ \\ ${ }^{1}$ Centre for Advanced Studies in Pure and Applied Mathematics, Bahauddin Zakariya University, Multan 60000, Pakistan \\ ${ }^{2}$ School of Mathematical Sciences, Zhejiang University, Hangzhou 310027, China \\ ${ }^{3}$ Zhejiang Province Key Laboratory of Quantum Technology and Device, Department of Physics, Zhejiang University, \\ Hangzhou 310027, China \\ ${ }^{4}$ Department of Mathematics, Huzhou University, Huzhou 313000, China \\ ${ }^{5}$ Hunan Provincial Key Laboratory of Mathematical Modeling and Analysis in Engineering, \\ Changsha University of Science \& Technology, Changsha 410114, China
}

Correspondence should be addressed to Yu-Ming Chu; chuyuming@zjhu.edu.cn

Received 24 March 2020; Accepted 24 June 2020; Published 3 August 2020

Academic Editor: Alessandro Contento

Copyright (C) 2020 Saima Akram et al. This is an open access article distributed under the Creative Commons Attribution License, which permits unrestricted use, distribution, and reproduction in any medium, provided the original work is properly cited.

In this article, approaches to estimate the number of periodic solutions of ordinary differential equation are considered. Conditions that allow determination of periodic solutions are discussed. We investigated focal values for first-order differential nonautonomous equation by using the method of bifurcation analysis of periodic solutions from a fine focus $\mathscr{Z}=0$. Keeping in focus the second part of Hilbert's sixteenth problem particularly, we are interested in detecting the maximum number of periodic solution into which a given solution can bifurcate under perturbation of the coefficients. For some classes like $C_{7,7}, C_{8,5}, C_{8,6}, C_{8,7}$, eight periodic multiplicities have been observed. The new formulas $\xi_{10}$ and $\varkappa_{10}$ are constructed. We used our new formulas to find the maximum multiplicity for class $C_{9,2}$. We have succeeded to determine the maximum multiplicity ten for class $C_{9,2}$ which is the highest known multiplicity among the available literature to date. Another challenge is to check the applicability of the methods discussed which is achieved by presenting some examples. Overall, the results discussed are new, authentic, and novel in its domain of research.

\section{Introduction}

Recently, the bifurcation analysis has attracted the attention of many researchers because it has wild applications in dynamics system and the universal existence of bifurcation in the nature, for example, bifurcation occurs when the small smooth change of parameters leads to qualitative change of its behavior (see [1]), aerodynamic limit cycle oscillation, and nonlinear oscillation in power system; the homoclinic and heteroclinic branches of a limit cycle colliding with one saddle point, two, or more saddle points and multiple biological dynamical systems are also bifurcation.

As nature is changing every moment and many changes occurring in nature are periodic like weather, blood flow inside body, circadian rhythm, oceans, and even human behavior, the study of theory of periodic or almost periodic solution is gaining attention. These periodic cycles are often observed to explore the impact of environmental factors in mathematical biology, food supplies, and harvesting. One of the classical mathematics problems is the theoretical calculation of the periodic solution of planar vector field.

The question about the number of periodic solution for nonautonomous differential equation continues to attract more interest. Neto in [2] states that for equation (1), we are unable to have upper bound for number of periodic solutions until some coefficients are restricted. Kadry in [3] gives conditions that allow determination for both upper and lower bounds. Our basic focus is to acquire highest periodic solutions of any class of the type (1); our main concern in this paper is this nearby question of bifurcation. Without loss of 
generality for above said arguments, we are considering the differential equation of the form

$$
\dot{\mathscr{Z}}=\gamma(\tau) \mathscr{Z}^{3}+\delta(\tau) \mathscr{Z}^{2}+v(\tau) \mathscr{Z},
$$

where independent variable $\tau$ and coefficients $\gamma, \delta, v$ are real valued functions but $\mathscr{Z} \in \mathbb{C}$. To find maximum number of periodic solutions, we use complexified form of equation (1) (for more details, see $[2,4-6]$ ). The above equation is the reminiscent of the equation described in Lloyd [5]:

$$
\dot{\mathscr{Z}}=p_{0}(\tau) \mathscr{Z}^{n}+p_{1}(\tau) \mathscr{Z}^{n-1}+p_{2}(\tau) \mathscr{E}^{n-2}+\cdots+p_{n}(\tau),
$$

with the assumption that $p_{0}(\tau)=1$ and $p_{1}(\tau), \ldots, p_{n}(\tau)$ were periodic functions having same period. It was shown that for $n=3$ in (2), there are three periodic solutions; also, the equation takes form as Abel's differential equation of first kind. It is important because of its connection with the wellknown Hilbert's sixteenth problem [7] for differential equations with polynomial coefficients:

$$
\left\{\begin{array}{l}
\dot{f}=\mathscr{F}(f, h), \\
\dot{h}=\mathscr{H}(f, h) .
\end{array}\right.
$$

Here, $\mathscr{F}$ and $\mathscr{H}$ are polynomials in $f$ and $h$. Then, Hilbert's sixteenth problem is transformed to polynomial equation, where leading coefficient randomly changes its sign.

For equation (1), complexified form is used so that we can take the maximum number of periodic solutions for each class using the perturbation method. For this reason, periodic solutions cannot be destroyed by any small perturbation of the coefficients. Consider that for equation (1) there exists $\beta \in \mathbb{R}$ such that

$$
\mathscr{E}(\beta)=\mathscr{E}(0) \text {. }
$$

These solutions are periodic, even if $\gamma, \delta$, and $v$ are not themselves periodic. Limit cycles bifurcate out of the fine focus $\mathscr{Z}=0$ when the coefficients of $\gamma$ and $\delta$ are slightly perturbed. By this method, the obtained limit cycles are said to be of small amplitude. The number of periodic solutions depends upon the multiplicity of solution $\mathscr{Z}=0$. The multiplicity of $\mathscr{Z}=0$ as a solution of equation (1) is also a multiplicity of the following displacement function:

$$
p: r \longrightarrow \mathscr{E}(\beta, 0, r)-r \text {. }
$$

For $\mathscr{Z}=0$, the method to compute multiplicity " $\mu$ " is explained in [4]; for the sake of ease, we explained it briefly here. We write $\mathscr{Z}(\tau, 0, r)=\sum_{i=1}^{\infty} \xi_{i}(\tau) r^{i}$ for $0 \leq \tau \leq \beta$, where $r$ lies in the regions near $\mathscr{Z}=0$, and use it in equation (5). For more details, see $[2,5,6,8]$ equation, which provide differential equation for $\xi_{\varkappa}(\tau)$, having some starting conditions $\xi_{1}(0)=1$ and $\xi_{\varkappa}(0)=0$ for $i>1$. Therefore,

$$
p(r)=\left(\xi_{1}(\alpha)-1\right) r+\sum_{i=2}^{\infty} \xi_{i}(\beta) r^{i} .
$$

The multiplicity $(x)$ is " $\varkappa>1$ " if

$$
\begin{aligned}
& \xi_{1}(\beta)=1, \\
& \xi_{2}(\beta)=\xi_{3}(\beta)=\ldots=\xi_{\varkappa-1}(\beta)=0 .
\end{aligned}
$$

However, $\xi_{\varkappa}(\beta) \neq 0$. When $\xi_{1}(\beta)=1$ and $\xi_{\varkappa}(\beta)=0, \forall$ $x>1$ origin is the center. We can observe from equation (1) that $\dot{\xi}_{1}(\tau)=\xi_{1}(\tau) v(\tau)$, where $\xi_{1}(\tau)$ is defined as

$$
\xi_{1}(\tau)=e^{\int_{0}^{\tau} v(s) \mathrm{d} s} .
$$

In this way, $\mu>1$ if

$$
\int_{0}^{\tau} v(s) \mathrm{d} s=0 .
$$

Because $\xi_{1}(\tau)=1$, we are especially interested about situation when $\mathscr{Z}=0$ has multiple solutions. So, we consider that (9) holds by applying the following conversion:

$$
\xi=z e^{-\int_{0}^{\tau} v(s) \mathrm{d} s}
$$

Equation (1) takes the following form:

$$
\dot{\xi}=\widehat{\gamma}(\tau) \xi^{3}+\widehat{\delta}(\tau) \xi^{3},
$$

where $\hat{\gamma}(\tau)=\gamma(\tau) e^{2 \int_{0}^{\tau} v(s) \mathrm{d} s}$ and

$$
\widehat{\delta}(\tau)=\delta(\tau) e^{2 \int_{0}^{\tau} v(s) \mathrm{d} s} .
$$

We can see that $\hat{\gamma}$ and $\widehat{\delta}$ are periodic if $\gamma, \delta$, and $v$ are periodic. By using Lemma (2.6) in [4], we consider multiplicity of $\mathscr{Z}=0$ as periodic solution of (1); if for equation (1), $x>1$, then the multiplicity of $\xi=0$ as a periodic solution of (11) is also $\chi$. So, we consider that $v(\tau) \stackrel{\simeq}{=} 0$ in (1). As a result, equation (1) takes the form as

$$
\dot{\mathscr{Z}}=\gamma(\tau) \mathscr{Z}^{3}+\delta(\tau) \mathscr{Z}^{2} .
$$

Here $\gamma$ and $\delta$ may be polynomials (i) in $\tau$ and (ii) in $\cos t$ and $\sin t$ (trigonometric functions) (for more details, see $[4,9,10])$. The functions $\xi_{i}(\tau)$, for $i>1$ are calculated by utilizing the present relation:

$$
\dot{\xi}_{i}=\gamma \sum_{\substack{j+k+l=i \\ j, k, l \geq 1}} \xi_{j} \xi_{k} \xi_{l}+\delta \sum_{\substack{j+k=i \\ j, k \geq 1}} \xi_{j} \xi_{k},
$$

with $\xi_{1}(\tau)=1$. However, as " $i$ " increases, certain calculations become tremendously complicated because of integration by parts, used in it. Assume that $\varkappa_{i}=\xi_{i}(\beta)$; at that point, $\varkappa=i$ if $\varkappa_{1}=1$ and $\varkappa_{k}=0$ for $2 \leq k \leq i-2$, but $\varkappa_{i} \neq 0$, and these $\varkappa_{i, s}$ are known as focal values. For $i \leq 8$, functions $\xi_{i}(\tau)$ and $\varkappa_{i}$ are given in [4]; for $i=9$, Yasmin in [10] had calculated $\xi_{9}(\tau)$ and $\varkappa_{9}$. For $i=10$, we have calculated $\xi_{10}(\tau)$ and $\varkappa_{10}$ which are given in Section 2. In equation (11), we make restriction for $n \leq 10$. We do not give the complete detailed derivation because they form a complicated web.

In Section 2, we have discussed classical formulas by which we are able to calculate the maximum multiplicity. With the help of classical results by Alwash given in [4], we are able to define some conditions w.r.t stopping criteria for our equation (1), and some suitable perturbations are defined in Section 3. Sections 2 and 3 are mainly concerned for calculation of focal values, which are used in Section 4. In Section 4, calculation of focal values is done for equation (13) having polynomial coefficients. In Section 5, some examples are given, and in Section 6 , conclusions about periodic multiplicity are discussed. 


\section{Calculation of the Focal Values $\varkappa_{10}$}

For equation (14), the functions $\xi_{2}, \xi_{3}, \ldots, \xi_{8}$ are given in [4], whereas $\xi_{9}, \xi_{10}$ are present in following theorem.
Theorem 1. For equation (14), conclusive functions $\xi_{2}, \xi_{3}, \ldots, \xi_{8}$ are given in [4]. The function $\xi_{9}$ is calculated in [11] and we succeeded to calculate $\xi_{10}$ which are given below:

$\xi_{9}=\bar{\delta}^{8}+7 \bar{\delta}^{6} \bar{\gamma}+\overline{\bar{\delta}^{6} \gamma}+6 \bar{\delta} \overline{\bar{\delta} \gamma}+2 \overline{\bar{\delta}^{5} \gamma} \bar{\delta}+5 \bar{\delta}^{4} \overline{\bar{\delta}^{2} \gamma}+3 \overline{\bar{\delta}^{4} \gamma} \bar{\gamma}+3 \overline{\bar{\delta}^{3} \gamma} \bar{\delta}^{2}+5 \overline{\bar{\delta}^{4} \gamma \bar{\gamma}}+\frac{39}{2} \bar{\delta}^{4} \bar{\gamma}^{2}-2 \bar{\delta}^{3} \overline{\delta \bar{\gamma}^{2}}$

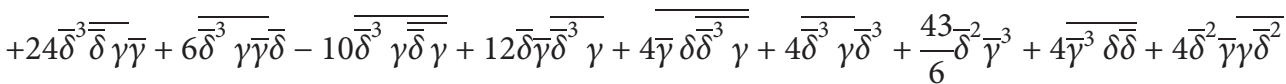

$-10 \overline{\delta \bar{\delta} \bar{\gamma} \overline{\gamma \bar{\delta}^{2}}}+\frac{15}{2} \bar{\gamma}^{2} \overline{\bar{\delta}^{2} \gamma}+2 \bar{\delta}^{2} \overline{\bar{\delta}^{2} \gamma}-2 \bar{\delta}^{4} \bar{\gamma}+8 \overline{\gamma \delta \bar{\delta}^{3}}+2 \overline{\delta \delta^{2} \gamma \overline{\bar{\delta} \gamma}}+26 \overline{\bar{\delta} \gamma \bar{\delta}^{2} \gamma \bar{\delta}}+6 \overline{\bar{\delta}^{2} \gamma \bar{\gamma}}-6 \overline{\bar{\delta}^{2} \gamma \bar{\gamma}}$

$+12 \bar{\delta}^{2} \overline{\bar{\delta} \gamma \bar{\gamma}}+16 \overline{\bar{\delta}^{2}} \gamma \delta \bar{\delta} \bar{\gamma}-16 \overline{\bar{\delta}^{3} \gamma \overline{\bar{\delta} \gamma}}+9 \bar{\delta}^{2} \overline{(\bar{\delta} \gamma)^{2}}+9 \overline{(\bar{\delta} \gamma)^{2}} \bar{\gamma}-\overline{\delta \bar{\gamma}^{3}} \bar{\delta}+\frac{35}{8} \bar{\gamma}^{4}-6 \bar{\delta} \bar{\gamma} \bar{\delta} \bar{\gamma}^{2}+6 \overline{\bar{\gamma}} \delta \bar{\gamma}^{2} \delta$

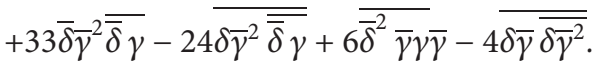

$\xi_{10}=\bar{\delta}^{9}-\frac{23}{2} \overline{\bar{\delta}^{7} \gamma}-\frac{1235}{6} \overline{\bar{\delta}^{5} \bar{\gamma} \gamma}+3 \overline{\bar{\delta}^{5} \gamma} \bar{\gamma}+111 \bar{\gamma} \bar{\delta}^{4} \overline{\bar{\delta} \gamma}-444 \bar{\gamma} \delta \overline{\delta^{3}} \overline{\bar{\delta} \gamma}+20 \bar{\gamma} \overline{\delta \delta^{4} \gamma}-12 \bar{\gamma} \delta \overline{\bar{\delta}} \overline{\bar{\delta}^{4}}+\frac{214}{3} \bar{\gamma} \bar{\delta}^{3} \overline{\bar{\delta}^{2} \gamma}+3 \gamma \bar{\delta}^{7}$

$-36 \overline{\left(\bar{\gamma}^{2} \delta \overline{\delta(\delta \bar{\gamma})}\right)}-48 \overline{\delta\left(\bar{\gamma}^{2} \delta \overline{(\bar{\delta} \gamma)}\right)}-16 \overline{\left(\gamma \bar{\delta} \overline{\left(\bar{\gamma} \bar{\delta}^{2} \gamma\right)}\right)}-8 \overline{\delta\left(\delta \bar{\gamma} \overline{\left(\delta \bar{\gamma}^{2}\right)}\right)}+8 \bar{\delta}^{2} \overline{\left(\bar{\delta}^{2} \gamma \delta \bar{\gamma}\right)}$

$-160 \bar{\gamma} \delta \bar{\delta}^{2} \overline{\bar{\delta}^{2} \gamma}+\frac{15}{2} \bar{\gamma}^{2} \overline{\bar{\delta}^{3} \gamma}-\frac{127}{2} \overline{\delta^{4} \gamma \overline{\bar{\delta} \gamma}}+30 \bar{\gamma} \bar{\delta}^{2} \overline{\bar{\delta}^{3} \gamma}-68 \bar{\gamma} \overline{\delta \overline{\delta \delta^{3} \gamma}}+24 \bar{\gamma} \overline{\bar{\delta}^{2} \gamma \delta \bar{\gamma}}+\frac{1015}{9} \bar{\gamma}^{3} \bar{\delta}^{3}$

$-237 \delta \overline{\delta \bar{\delta}^{2} \bar{\gamma}^{3}}+8 \bar{\gamma} \bar{\delta}^{7}-\frac{11}{2} \bar{\gamma} \bar{\delta}^{2} \overline{\delta \bar{\gamma}^{2}}+26 \overline{\gamma \bar{\gamma} \bar{\delta} \overline{\delta \bar{\gamma}^{2}}}+\frac{319}{2} \bar{\gamma}^{2} \bar{\delta}^{2} \bar{\delta} \gamma-174 \delta \overline{\delta \bar{\gamma} \bar{\gamma}^{2} \overline{\bar{\delta} \gamma}}-90 \bar{\gamma} \overline{\overline{\delta \delta} \bar{\delta}^{2}}+9 \bar{\gamma} \overline{\bar{\delta}^{3} \gamma \bar{\gamma}}$

$+40 \bar{\gamma} \bar{\delta} \overline{\gamma \bar{\gamma} \bar{\delta}^{2}}-24 \bar{\gamma} \delta \overline{\gamma \bar{\gamma} \bar{\delta}^{2}}+3 \overline{\bar{\gamma} \bar{\delta}^{2} \gamma \overline{\bar{\delta} \gamma}}-154 \bar{\gamma} \gamma \bar{\delta}^{2} \bar{\delta} \gamma-24 \overline{\gamma \bar{\gamma}^{2} \bar{\delta}^{2} \delta}+24 \gamma \bar{\gamma}^{2} \overline{\delta \delta^{2} \gamma}+42 \bar{\gamma} \delta(\overline{\bar{\delta} \gamma})^{2}-5 \gamma \bar{\delta} \bar{\delta}^{6}$

$-\frac{3}{2} \bar{\gamma} \overline{\delta \bar{\gamma}^{3}}-21 \overline{\delta \bar{\gamma}^{4}}+\overline{\delta \overline{\bar{\delta} \gamma} \overline{\delta \bar{\gamma}^{2}}}-\frac{15}{4} \bar{\gamma}^{2} \overline{\delta \bar{\gamma}^{2}}+\frac{169}{4} \bar{\gamma}^{4} \bar{\delta}+70 \bar{\gamma} \bar{\delta}(\overline{\bar{\delta} \gamma})^{2}-2 \overline{\gamma \bar{\delta} \overline{\bar{\delta}^{4} \gamma}}+10 \bar{\gamma}^{3} \overline{\bar{\delta} \gamma}+\frac{9}{2} \bar{\delta}^{4} \overline{\bar{\delta}^{3} \gamma}$

$-74 \overline{\gamma \bar{\gamma}^{3} \bar{\delta}}+8 \bar{\delta} \overline{\delta \bar{\delta} \bar{\gamma}^{3}}+7 \bar{\delta}^{6} \overline{\bar{\delta} \gamma}+\frac{1}{2} \bar{\delta}^{4} \overline{\bar{\delta} \gamma}+2 \overline{\delta \delta^{6} \gamma}-16 \bar{\gamma} \delta \bar{\delta}^{4}-70 \bar{\gamma} \bar{\delta}^{3} \gamma^{2}+6 \bar{\delta}^{5} \overline{\bar{\delta}^{2} \gamma}-6 \overline{\gamma \bar{\delta} \overline{\delta^{4}} \gamma}+2 \bar{\delta}^{3} \overline{\bar{\delta}^{3} \gamma}$

$+10 \bar{\delta} \overline{\gamma \bar{\gamma} \bar{\delta}^{4}}+26 \bar{\gamma}^{2} \bar{\delta}^{5}-\frac{5}{2} \bar{\delta}^{4} \overline{\delta \bar{\gamma}^{2}}+\frac{5}{2} \overline{\delta \bar{\delta}^{4} \bar{\gamma}^{2}}+\frac{73}{2} \bar{\gamma} \bar{\delta}^{4} \bar{\delta} \gamma+16 \overline{\delta \delta^{3} \bar{\gamma} \delta}+9 \bar{\delta}^{2} \overline{\gamma \bar{\gamma} \bar{\delta}^{3}}-\frac{4}{5} \bar{\gamma} \bar{\delta}^{5}+12 \bar{\gamma} \overline{\delta \delta^{2} \gamma}$

$-21 \overline{\bar{\delta}^{2} \gamma \overline{\bar{\delta}^{3} \gamma}}+8 \overline{\delta \bar{\gamma} \delta \overline{\bar{\delta}^{3} \gamma}}-\frac{160}{3} \overline{\gamma \bar{\delta}^{3} \overline{\bar{\delta}^{2} \gamma}}+19 \gamma \bar{\gamma}^{2} \overline{\bar{\delta}^{3} \gamma}+32 \overline{\bar{\delta} 4 \overline{\gamma \delta \bar{\gamma}}}-20 \overline{\delta \bar{\gamma} \bar{\delta} \delta \overline{\bar{\delta}^{2} \gamma}}+24 \bar{\delta} \bar{\gamma}^{2} \overline{\bar{\delta}^{2} \gamma}+\frac{4}{3} \bar{\delta}^{3} \overline{\bar{\delta}^{2} \gamma}$

$-\frac{31}{30} \gamma \overline{\bar{\delta}^{5}}-\frac{970}{3} \gamma \bar{\gamma}^{2} \bar{\delta}^{3}+12 \overline{\delta \delta \bar{\gamma}^{2}} \overline{\delta \bar{\gamma}}+\frac{13}{2} \overline{\bar{\delta}^{2} \gamma \overline{\delta \gamma^{2}}}+3 \bar{\delta}^{2} \overline{\bar{\delta}^{2} \gamma \overline{\bar{\delta} \gamma}}+42 \bar{\delta}^{2} \overline{\bar{\delta}^{2} \gamma} \overline{\bar{\delta} \gamma}-20 \overline{\delta \delta^{3} \gamma \overline{\bar{\delta} \gamma}}-12 \overline{\gamma \bar{\delta} \overline{\bar{\delta}^{2} \gamma}}$

$-12 \overline{\delta \bar{\delta}^{2} \bar{\gamma} \gamma}+12 \bar{\delta}^{3} \overline{\bar{\delta}^{2} \bar{\gamma} \gamma}+32 \overline{\delta^{2} \bar{\gamma} \overline{\delta \bar{\delta}^{2} \gamma}}-32 \overline{\delta \delta^{3} \gamma \overline{\delta \bar{\gamma}}}+14 \bar{\delta}^{3}(\overline{\bar{\delta} \gamma})^{2}-28 \gamma \bar{\delta}(\overline{\bar{\delta} \gamma})^{2}-\frac{3}{2} \bar{\delta}^{2} \overline{\delta \bar{\gamma}^{3}}-15 \overline{\bar{\delta}^{5} \gamma^{2}}$

$-8 \overline{\delta \bar{\delta}^{4} \gamma \bar{\gamma}}-24 \overline{\bar{\gamma}^{2} \delta \overline{\bar{\delta}^{2} \gamma}}+\frac{34}{3} \bar{\gamma} \bar{\delta}^{3} \overline{\bar{\delta}^{2} \gamma}+2 \overline{\delta \bar{\delta}} \bar{\gamma} \overline{\bar{\delta}^{3} \gamma}+\bar{\delta}\left(\overline{\bar{\delta}^{2} \gamma}\right)^{2}$. 
By using these $\xi_{i}$ functions, we get Theorem 2 that enables us to find the maximum multiplicity in which integral is like $\int \gamma(\tau) \overline{\delta(\tau)} \mathrm{d} \tau$ and bar "-" shows integral $\overline{\delta(\tau)}=\int_{0}^{\tau} \delta(\tau) \mathrm{d} \tau$, which is definite.
Theorem 2. The solution $\mathscr{Z}=0$ of (13) has a multiplicity $k$, wherever $2 \leq k \leq 10$ if $\varkappa_{n}=0$ for $2 \leq n \leq k-1$ and $\varkappa_{n} \neq 0$ where

$$
\begin{aligned}
& \varkappa_{2}=\int_{0}^{\beta} \delta \text {, } \\
& x_{3}=\int_{0}^{\beta} \gamma \\
& \varkappa_{4}=\int_{0}^{\beta} \gamma \bar{\delta} \\
& \varkappa_{5}=\int_{0}^{\beta} \gamma \bar{\delta}^{2} \\
& \varkappa_{6}=\int_{0}^{\beta} \gamma \bar{\delta}^{3}-\frac{1}{2} \bar{\delta} \bar{\gamma} \delta \\
& \varkappa_{7}=\int_{0}^{\beta} \gamma \bar{\delta}^{4}+2 \gamma \bar{\delta}^{2} \bar{\gamma} \\
& \varkappa_{8}=\int_{0}^{\beta} \gamma \bar{\delta}^{5}+3 \gamma \bar{\delta}^{3} \bar{\gamma}+\gamma \bar{\delta}^{2} \overline{\bar{\delta} \gamma}-\frac{1}{2} \bar{\gamma}^{3} \delta \\
& \varkappa_{9}=\int_{0}^{\beta} \gamma \bar{\delta}^{6}-5 \gamma \bar{\delta}^{4} \bar{\gamma}-2 \bar{\delta}^{3} \overline{\delta \bar{\gamma}}+20 \overline{\delta \bar{\gamma}^{2}}+2 \overline{\delta \bar{\gamma}} \delta \bar{\gamma}^{2} \text {, } \\
& \varkappa_{10}=\int_{0}^{\beta} \gamma \bar{\delta}^{7}-\frac{1235}{6} \gamma \bar{\gamma} \bar{\delta}^{5}-\frac{970}{3} \gamma \bar{\gamma}^{2} \bar{\delta}^{3}-237 \delta \bar{\delta}^{2} \bar{\gamma}^{3}-24 \gamma \bar{\gamma}^{2} \delta \bar{\delta}^{2} \\
& -70 \bar{\gamma} \bar{\delta}^{3} \gamma^{2}-21 \bar{\gamma}^{4} \delta-74 \gamma \bar{\gamma}^{3} \bar{\delta}+\frac{5}{2} \bar{\gamma}^{2} \delta \bar{\delta}^{4}+32 \bar{\delta}^{4} \gamma \overline{\delta \bar{\gamma}}-16 \delta \bar{\delta}^{4} \bar{\gamma}-15 \bar{\delta}^{5} \gamma^{2} \\
& -36 \delta \bar{\delta} \bar{\gamma}^{2} \bar{\delta} \bar{\gamma}-8 \delta \bar{\delta}^{4} \gamma \bar{\gamma}
\end{aligned}
$$

\section{Conditions for Center and Method of Perturbation}

In this section, we describe some conditions for center. From Theorem 2 , we find maximum value $\varkappa$ for different classes of nonautonomous equation of the form as (1). Stopping criteria are defined for calculating maximum multiplicity $\varkappa_{k}$. We need some conditions that assure that there is no need to proceed further $\left(\varkappa_{k}\right)$. For this, suitable conditions that are sufficient for $\mathscr{Z}=0$ as a center are given below in the form of theorems and corollaries.

Theorem 3. Consider that there are continuous functions $f$, $g$ defined on $I=\sigma([0, \alpha])$ and differentiable function $\sigma$ with $\sigma(\alpha)=\sigma(0)$ such that

$$
\begin{aligned}
& \gamma(\tau)=f(\sigma(\tau)) \dot{\sigma} \\
& \delta(\tau)=g(\sigma(\tau)) \dot{\sigma}
\end{aligned}
$$

Then, the origin is a center for (13).

Theorem 4. The origin is a center for equation (13) if $\gamma$ is a constant multiple of $\delta$ and $\int_{0}^{\beta} \delta(\tau) d t=0$.

Corollary 1. If any $\delta$ or $\gamma$ is identically 0 and other has mean value zero, then the origin is a center.
After determining the maximum multiplicity $\mathcal{x}$, we have to make series of perturbation of the coefficients, every one of which results in one periodic solution to come out of origin. For more details, see $[4,5,12]$.

For this, suppose equation of the form given below:

$$
\dot{\mathscr{Z}}=\gamma(\tau) \mathscr{Z}^{3}+\delta(\tau) \mathscr{Z}^{2},
$$

having multiplicity $\varkappa=j$ (suppose). Let $\mathcal{U}$ be in the regions near 0 in the complex plane containing no periodic solutions except $\mathscr{Z}=0$. From Theorem (2.4) in [4], the initial point which is contained in $\mathcal{U}$ remained fixed with respect to total number of periodic solutions with the restriction that perturbation of the coefficients considered remained small enough. Our goal is to get $\varkappa_{2}=\varkappa_{3}=\ldots=\varkappa_{j-2}=0$ but $\varkappa_{j-1} \neq 0$ by perturbing and making suitable choices of $\gamma$ and $\delta$, if possible. Obviously, the most effective solutions in $\mathcal{U}$ and $\psi$ are zero solution while we get periodic solutions $\psi(\tau)$ where $\psi(0) \in \mathcal{U}$ as nontrivial solutions. By considering the underlying fact that the complex solutions always appear in conjugate pair, we can say that $\psi$ is real. Let $\mathcal{U}_{1}$ and $\mathscr{V}_{1}$ be the neighborhood of zero and $\psi$, respectively, such that $\mathscr{V}_{1} \cup \mathcal{U}_{1} \subset \mathcal{U}$ and $\mathscr{V}_{1} \cap \mathcal{U}_{1}=\phi$. The periodic solutions around $\mathscr{V}_{1}$ and $\mathscr{U}_{1}$ are preserved when we take small perturbation in the coefficients. By applying the same procedure as above, our choice is to perturb the coefficients such that $\varkappa_{k}=0$ for $k=$ $2,3, \ldots, j-3$ but $\varkappa_{j} \neq 0$. So, we get $x=j-2$. By applying 
that procedure, we get two nontrivial real periodic solutions with zero solution having multiplicity $j-2$. In this way, we end up with equation (18) having $\varkappa=2$ and $j-2$ distinct nontrivial (other than zero) real periodic solutions.

\section{Polynomial Coefficients for Some Classes}

For the polynomial " $\tau$," consider $C_{p, q}$ indicates the class of equation of the form (13) with degree $p$ and $q$ for $\gamma$ and $\delta$, respectively. We have evaluated the maximum multiplicity denoted by the symbol " $\mu$ " for some classes like $C_{7,7}, C_{8,5}$, $C_{8,6}, C_{8,7}$, and $C_{9,2}$ that are presented below in the form of theorems. For more classes with maximum multiplicity, see [9].

Theorem 5. Let $C_{9,2}$ be class of equation of form (13), with

$$
\begin{aligned}
& \gamma(\tau)=a+b \tau+c \tau^{2}+d \tau^{3}+f \tau^{5}+j \tau^{9} . \\
& \delta(\tau)=k+m \tau^{2} .
\end{aligned}
$$

Then, we conclude $\mu_{\max }\left(C_{9,2}\right) \geq 10$.

Proof. Using Theorem 2, we take

$$
\begin{aligned}
& \varkappa_{2}=k+\frac{1}{3} m, \\
& \varkappa_{3}=a+\frac{1}{2} b+\frac{1}{3} c+\frac{1}{4} d+\frac{1}{6} f+\frac{1}{10} j .
\end{aligned}
$$

Thus, multiplicity of $\mathscr{Z}=0$ is $x=2$ if $x_{2} \neq 0$. And multiplicity is $x=3$ if $\varkappa_{2}=0$ but $x_{3} \neq 0$. If $\varkappa_{2}=\varkappa_{3}=0$, then by using value of " $k$ " and " $a, " \gamma(\tau)$ and $\delta(\tau)$ are as follows:

$$
\begin{aligned}
\gamma(\tau)= & b\left(\tau-\frac{1}{2}\right)+c\left(\tau^{2}-\frac{1}{3}\right)+d\left(\tau^{3}-\frac{1}{4}\right) \\
& +f\left(\tau^{5}-\frac{1}{6}\right)+j\left(\tau^{9}-\frac{1}{10}\right), \\
\delta(\tau)= & m\left(\tau^{2}-\frac{1}{3}\right) .
\end{aligned}
$$

And we compute $\varkappa_{4}$ as given below:

$$
\varkappa_{4}=-\frac{m(-7938 j-7150 f-3861 d+6006 b)}{2162160} .
$$

If $\varkappa_{4}=0$, then either $m=0$ or

$$
j=-\frac{7150}{7938} f-\frac{3861}{7938} d+\frac{6006}{7938} b .
$$

If $m=0$, then $\delta(\tau)=0$ and $\varkappa_{3}=0$ gives that mean value of $\gamma(\tau)$ is zero. Thus, origin is a center from Corollary 1 . So, consider $m \neq 0$. Now, if (24) holds, then we calculate

$$
\varkappa_{5}=\frac{m^{2}(-4070 f-2727 d+1302 b)}{200037600} .
$$

If $\varkappa_{5}=0$, we consider $m \neq 0$ which implies that

$$
f=-\frac{2727}{4070} d+\frac{1302}{4070} b \text {. }
$$

And by using (26), we take $x_{6}$ as

$$
\varkappa_{6}=-\frac{m(d+4 b)\left(43475961 b+47732590 m^{2}-7030731 d\right)}{4535399964595500} .
$$

If $\varkappa_{6}=0$, we consider $m \neq 0$ and either $d=-4 b$ or

$$
b=-\frac{47732590}{43475961} m^{2}+\frac{7030731}{43475961} d .
$$

If $d=-4 b$, then (26) gives $f=3 b$, and (24) gives $j=0$. By using values of $d, f, j$, equations (21) and (22) take the following form:

$$
\begin{aligned}
& \gamma(\tau)=c\left(\tau^{2}-\frac{1}{3}\right)+b\left(3 \tau^{3}-3 \tau\right)\left(\tau^{2}-\frac{1}{3}\right), \\
& \delta(\tau)=m\left(\tau^{2}-\frac{1}{3}\right) .
\end{aligned}
$$

Let $\sigma(\tau)=\tau^{3}-\tau ; \quad$ then, $\quad \dot{\sigma}(\tau)=3 \tau^{2}-1 . \quad$ Also, $\sigma(0)=\sigma(1)$. So, we write above equations as

$$
\begin{aligned}
& \gamma(\tau)=\frac{1}{3}\left[c+b\left(3 \tau^{3}-3 \tau\right)\right] \dot{\sigma}, \\
& \delta(\tau)=\frac{m}{3} \dot{\sigma} .
\end{aligned}
$$

Thus, from Theorem 3, origin is the center with

$$
f(\sigma)=\frac{1}{3}\left[c+b\left(3 \tau^{3}-3 \tau\right)\right]
$$

and $g(\sigma)=m / 3$. So, we take $d \neq-4 b$. If (28) holds, then we compute $\varkappa_{7}$ as

$$
\varkappa_{7}=-\frac{129007 m^{2}\left(-8 m^{2}+3 d\right)\left(244401908873421 d-51374543768976 m^{2}+343350184670592 c\right)}{5750115960595749963075535680} .
$$

If $\varkappa_{7}=0$, recalling that $m \neq 0$, then either $d=(8 / 3) m^{2}$ or

$$
\begin{aligned}
d= & \frac{513745143768976}{244401908873421} m^{2} \\
& -\frac{343350184670592}{244401908873421} c .
\end{aligned}
$$

If $d=(8 / 3) m^{2}$, then

$$
\begin{aligned}
& \gamma(\tau)=\frac{1}{3}\left[c+m^{2}\left(-2 \tau^{3}+2 \tau\right)\right] \dot{\sigma}, \\
& \delta(\tau)=\frac{m}{3} \dot{\sigma} .
\end{aligned}
$$

From Theorem 3, origin is the center with $f(\sigma)=(1 / 3)\left[c+m^{2}\left(-2 \tau^{3}+2 \tau\right)\right]$ and $g(\sigma)=(m / 3)$. So, consider $d \neq(8 / 3) m^{2}$. By using (33), we calculate $\varkappa_{8}$ as 


$$
\varkappa_{8}=\frac{5609 m\left(269232 c+108205 m^{2}\right) \oplus}{464011623410213337535103836105743901025559739592193301950},
$$

where

$$
\begin{aligned}
\oplus= & 4403049224583165170231259327919284 c^{2} \\
& -4081167090232676699163689650491745 \mathrm{~cm}^{2} \\
& -79907221802386910423708643546694695176 \mathrm{~m}^{4} .
\end{aligned}
$$

Now, if $x_{8}=0$, then either $\omega=0$ or

$$
c=-\frac{108205}{269232} m^{2}
$$

because $m \neq 0$. If (37) holds but $₫ \neq 0, m \neq 0$, then we compute $\varkappa_{9}$ as

$$
\varkappa_{9}=-\frac{m^{5}(7692367147318 m+641819984880883)}{3796688520035034624} .
$$

If equation $(37) \neq 0, \quad l \neq 0$, but $\omega=0$ holds, then $c=v_{i} m^{2} / 3$ for $i=1,2$ with $v_{1}=45.009923140$ and $v_{2}=-17.20305420$. If $\varkappa_{9}=0$ recalling that $m \neq 0$, we take value of $m$ as

$$
m=-\frac{641819984880883}{7692367147318}
$$

If (39) holds, then we calculate $\varkappa_{10}$ as

$$
\begin{array}{r}
2361993032553605726067964864810817973318238075190243974713862267553689045303 \\
\varkappa_{10}=-\frac{4093572801545113943979704942365450213502975589695413549237735086533209175509}{1544470146002546211838899443514363633204787915211352260941096546167381} \\
93865483843471924056732079968557936379195349112486049662164194337751040 .
\end{array}
$$

Here $\varkappa_{10}$ is equal to constant number which is nonzero. Hence, we conclude that multiplicity of class $C_{9,2}$ is 10 , i.e., $\mu_{\max }\left(C_{9,2}\right) \geq 10$.

Theorem 6. Consider the equation given below:

$$
\dot{\mathscr{Z}}=\gamma(\tau) \mathscr{Z}^{3}+\delta(\tau) \mathscr{Z}^{2},
$$

$$
\begin{aligned}
\gamma(\tau)= & \frac{108205}{807696}\left(-\frac{641819984880883}{7692367147318}+\varepsilon_{1}\right)^{2}+\frac{1124460226139}{81467302957807} \varepsilon_{2}-\frac{118170412}{478235571} \varepsilon_{3}-\frac{3664}{6105} \varepsilon_{4} \\
& -\frac{304}{39} \varepsilon_{5}-\frac{1}{10} \varepsilon_{6}+\varepsilon_{7}+\left(-\frac{2}{3}\left(-\frac{641819984880883}{7692367147318}+\varepsilon_{1}\right)^{2}-\frac{18508333737344}{81467302957807} \varepsilon_{2}+\frac{2343577}{14491987} \varepsilon_{3}+\varepsilon_{4}\right) t \\
& +\left(-\frac{108205}{269232}\left(-\frac{641819984880883}{7692367147318}+\varepsilon_{1}\right)^{2}+\varepsilon_{2}\right) t^{2} \\
& +\left(\frac{8}{3}\left(-\frac{641819848083}{769236147318}+\varepsilon_{1}\right)^{2}-\frac{1144506156864}{8146730297807} \varepsilon_{2}+\varepsilon_{3}\right) t^{3}+\left(\left(-\frac{6418198480883}{7692367147318}+\varepsilon_{1}\right)^{2}\right. \\
& \left.+\frac{28649125056}{32982713481} \varepsilon_{2}+\frac{651}{2035} \varepsilon_{4}-\frac{197126007}{318823714} \varepsilon_{3}+\varepsilon_{5}\right) t^{5} \\
& +\left(-\frac{509145851520}{188014636417} \varepsilon_{2}+\frac{838455}{4345961} \varepsilon_{3}+\frac{52}{111} \varepsilon_{4}-\frac{3575}{3969} \varepsilon_{5}+\varepsilon_{6}\right) t^{9} . \\
\delta(\tau)= & \frac{641819984880883}{23077101441954}-\frac{1}{3} \varepsilon_{1}+\varepsilon_{8}+\left(-\frac{641819984880883}{7692367147318}+\varepsilon_{1}\right) t^{2}
\end{aligned}
$$


Choose $\varepsilon_{p}$ for $1 \leq p \leq 8$ to be nonzero and small as compared to $\varepsilon_{p-1}$. Then, there exist eight real nontrivial periodic solutions for equation (41).

Proof. If we substitute $\varepsilon_{r}=0, \forall r=1,2, \ldots, 8$ and coefficients are as written above, then the multiplicity of the origin is 10 . Choose $\varepsilon_{1} \neq 0$ and $\varepsilon_{r}=0$ for $2 \leq r \leq 8$; it can be easily seen that $\varkappa_{9}$ is constant multiple of $\epsilon_{1}$ but $\varkappa_{2}=x_{3}=\ldots=x_{7}=x_{8}=0$, so the multiplicity reduces by one, and $x=9$. For that reason one periodic solution bifurcates out of the origin. Now, set $\epsilon_{1} \neq 0, \epsilon_{2} \neq 0$ and $\epsilon_{r}=0$ for $3 \leq r \leq 8$, and we have $\varkappa_{r}=0$ for $r=2,3, \ldots, 7$ but $\varkappa_{8}$ results in form of $\epsilon_{2}$ with some constant multiple, so $\varkappa=8$. Now, set $\varepsilon_{1} \neq 0, \varepsilon_{2} \neq 0, \varepsilon_{3} \neq 0$ and $\varepsilon_{r}=0$ for $4 \leq r \leq 8$; then, we have $\varkappa_{r}=0$ for $r=2,3, \ldots, 6$ but $\varkappa_{7}$ results in form of $\epsilon_{3}$ with some constant multiple. If $\epsilon_{2}$ is sufficiently small, then there are two nontrivial real periodic solutions. Further continuing the same procedure, we own eight real periodic nontrivial solutions.

\section{Corollary 2. For an equation}

$$
\dot{\mathscr{Z}}=\gamma(\tau) \mathscr{Z}^{3}+\delta(\tau) \mathscr{Z}^{2}+\tau+v \text {. }
$$

If $\gamma(\tau)$ and $\delta(\tau)$ are as given in Theorem 6 and $\tau$ and $v$ are small enough, equation (43) has ten real periodic solutions.

Proof. Given that $\tau=0, v=0$, and $\varkappa=2$, then (43) has eight real periodic solutions. If $\tau \neq 0$ but small enough, then $\varkappa=1$; using the same arguments as above, we have nine periodic solutions. These are distinct and other than $0, \mathscr{Z}=0$ is another such solution, and thus we take ten real periodic solutions.

Theorem 7. Consider $C_{8, j}$ as the class of equations of form (13) having degree 8 for $\gamma(\tau)$ and $j$ for $\delta(\tau)$; here $j=5,6,7$. Then, we cease with the results $\mu_{\max }\left(C_{8, j}\right) \geq 8$, for all $j=5,6,7$, respectively.

Proof. We first consider the class in which degree of $\gamma(\tau)$ is 7 , i.e., $C_{8,7}$. The classes in which degree of $\gamma(\tau)$ is less than 7 are the special cases. Let

$$
\left.\begin{array}{l}
\gamma(\tau)=a+b \tau+c \tau^{2}+d \tau^{3}+e \tau^{4}+f \tau^{5}+g \tau^{6}+h \tau^{7}+i \tau^{8} \\
\delta(\tau)=k+l \tau+m \tau^{2}+n \tau^{3}+o \tau^{4}+p \tau^{5}+q \tau^{6}+r \tau^{7}
\end{array}\right\} .
$$

By using Theorem 2, we calculate

$$
\begin{aligned}
& \varkappa_{2}=k+\frac{l}{2}+\frac{m}{3}+\frac{n}{4}+\frac{o}{5}+\frac{p}{6}+\frac{q}{7}+\frac{r}{8}, \\
& \varkappa_{3}=a+\frac{b}{2}+\frac{c}{3}+\frac{d}{4}+\frac{e}{5}+\frac{f}{6}+\frac{g}{7}+\frac{h}{8}+\frac{i}{9} .
\end{aligned}
$$

If $\varkappa_{2}=\varkappa_{3}=0$, we calculate $\varkappa_{4}$ as

$$
\begin{aligned}
\varkappa_{4}= & -\frac{1}{360} b m+\frac{1}{360} c l+\frac{1}{240} d l-\frac{1}{240} b n+\frac{1}{210} e l-\frac{1}{560} c n+\frac{1}{560} d m-\frac{1}{210} b o \\
& +\frac{5}{1008} f l-\frac{1}{360} c o+\frac{1}{360} e m-\frac{5}{1008} b p+\frac{5}{1008} g l+\frac{5}{1512} f m+\frac{1}{900} e n-900 d o \\
& -\frac{5}{1512} c p-\frac{5}{1008} b q+\frac{7}{1440} h l+\frac{1}{280} g m+\frac{1}{560} f n-\frac{7}{1440} b r-\frac{1}{560} d p \\
& -\frac{1}{280} c q \frac{5}{1092} g p+\frac{8}{4725} i o-\frac{5}{608} f r+\frac{5}{6048} h p+\frac{1}{2880} h q-\frac{1}{2880} g r+\frac{1}{1680} i q+\frac{7}{27540} i r .
\end{aligned}
$$

From $\varkappa_{4}$, we cannot substitute any value to proceed further. As a result, we make restriction of the coefficients for class $C_{8,7}$ like $c=d=f=g=l=m=n=o=p=q=0$ in the system of equation (44).

(1) Let

$$
\begin{aligned}
& \gamma(\tau)=a+b \tau+e \tau^{4}+h \tau^{7}+i \tau^{8}, \\
& \delta(\tau)=k+r \tau^{7} .
\end{aligned}
$$

Utilizing Theorem 2, we get

$$
\begin{aligned}
& \varkappa_{2}=k+\frac{r}{8}, \\
& \varkappa_{3}=a+\frac{b}{2}+\frac{e}{5}+\frac{h}{8}+\frac{i}{9} .
\end{aligned}
$$

Thus, multiplicity of $\mathscr{Z}=0$ is $x=2$ if $x_{2} \neq 0$. And multiplicity $x=3$ if $x_{2}=0$ but $x_{3} \neq 0$. If $x_{2}=x_{3}=0$, then we calculate $\varkappa_{4}$ as

$$
\varkappa_{4}=-\frac{7 r(-104 i+612 e+1989 b)}{2864160} \text {. }
$$


If $\varkappa_{4}=0$, then either $r=0$ or

$$
i=\frac{612}{104} e+\frac{1989}{104} b \text {. }
$$

If $r=0$, then $\varkappa_{2}=0$ gives $k=0$; hence, $\delta(\tau)=0$. If $\varkappa_{2}=0$, it means that the mean value of $\gamma(\tau)$ is zero. By Corollary 1 , origin is a center. Suppose that $r \neq 0$. By using (50), we compute

$$
\varkappa_{5}=-\frac{r^{2}(-768 e+31213 b)}{980179200} .
$$

If $\varkappa_{5}=0$, then

$$
e=\frac{31213}{768} b
$$

We have already taken $r \neq 0$. If (52) holds, then

$$
\varkappa_{6}=\frac{343 b r\left(2809107 r^{2}+142686496 b\right)}{836532043776000} \text {. }
$$

If $\varkappa_{6}=0$, then either $b=0$ or

$$
b=-\frac{2809107}{142686496 b} r^{2}
$$

because $r \neq 0$. If $b=0$, then from (50) and (52), $e=0, i=0$, respectively. By using these values, $\gamma(\tau)$ and $\delta(\tau)$ take the form as

$$
\begin{aligned}
& \gamma(\tau)=k\left(\tau^{7}-\frac{1}{8}\right), \\
& \delta(\tau)=r\left(\tau^{7}-\frac{1}{8}\right) .
\end{aligned}
$$

Let $\quad \sigma(\tau)=\tau^{8}-\tau ; \quad$ then, $\quad \dot{\sigma}(\tau)=8 \tau^{7}-1 ; \quad$ also, $\sigma(0)=\sigma(1)$. So, we can write as

$$
\begin{aligned}
& \gamma(\tau)=\frac{1}{8} k \dot{\sigma} \\
& \delta(\tau)=\frac{1}{8} r \dot{\sigma}
\end{aligned}
$$

Utilizing Theorem 3, origin is the center with $f(\sigma)=$ $(1 / 8) k$ and $g(\sigma)=(1 / 8) r$. So, we take $b \neq 0$. If (54) holds, then $\varkappa_{7}$ is given by

$$
\varkappa_{7}=-\frac{749407323 r^{4}\left(-31704452399633263173 r^{2}+5552181576645742592 h\right)}{137618755815002650025303511924736000} .
$$

Now if $\varkappa_{7}=0$, recalling that $r \neq 0$, then

Holding (58), we have

$$
h=\frac{31704452399633263173}{5552181576645742592} r^{2} .
$$

$$
\varkappa_{8}=\frac{108510679237512699726452487222116531317347537}{1176413089188756096613789685106805706496326217236480000} r^{7}
$$

That is, constant multiple of $r^{7}$ and $r$ is also nonzero. Thus, we conclude that multiplicity of class $C_{8,7}$ is 8 , i.e., $\mu_{\max }\left(C_{8,7}\right) \geq 8$.

(2) For the class $C_{8,6}$, the degree of $\delta(\tau)=6$ and $\gamma(\tau)=8$; we suppose that $c=d=f=h=l=m=$ $n=o=p=r=0$ in system of equation (44). Let

$$
\begin{aligned}
& \gamma(\tau)=a+b \tau+e \tau^{4}+g \tau^{6}+i \tau^{8}, \\
& \delta(\tau)=k+q \tau^{6} .
\end{aligned}
$$

By using Theorem 2, we calculate

$$
\begin{gathered}
\varkappa_{2}=k+\frac{q}{7} \\
\varkappa_{3}=a+\frac{b}{2}+\frac{e}{5}+\frac{g}{7}+\frac{i}{9} .
\end{gathered}
$$

Thus, multiplicity of $\mathscr{Z}=0$ is $x=2$ if $x_{2} \neq 0$. And multiplicity $x=3$ if $x_{2}=0$ but $x_{3} \neq 0$. If $x_{2}=\varkappa_{3}=0$, then 


$$
\begin{aligned}
& a=-\frac{b}{2}-\frac{e}{5}-\frac{g}{7}-\frac{i}{9} \\
& k=-\frac{q}{7}
\end{aligned}
$$

By using these values from (62) and (63), we have

$$
\begin{aligned}
& \gamma(\tau)=b\left(\tau-\frac{1}{2}\right)+e\left(\tau^{4}-\frac{1}{5}\right)+g\left(\tau^{6}-\frac{1}{7}\right)+i\left(\tau^{8}-\frac{1}{9}\right) \\
& \delta(\tau)=q\left(\tau^{6}-\frac{1}{7}\right) .
\end{aligned}
$$

We calculate $\varkappa_{4}$ as

$$
\varkappa_{4}=-\frac{q(-3 i+6 e+25 b)}{5040} .
$$

If $\varkappa_{4}=0$, then either $q=0$ or

$$
i=\frac{6}{3} e+\frac{25}{3} b \text {. }
$$

If $q=0$, then (63) gives $k=0$, so $\delta(\tau)=0$. For $\varkappa_{2}=0$, the mean value of $\gamma(\tau)$ is zero. So, by Corollary 1 , origin is the center. Consider that $q \neq 0$; if (50) holds, then $\varkappa_{5}$ is

$$
\varkappa_{5}=-\frac{q^{2}(-44399616 e+1080979445 b)}{35418224041200} .
$$

If $\varkappa_{5}=0$, then either $q=0$ or $e=(1080979445 /$ $44399616) b$. But as we have already seen that $q \neq 0$, by using value of $e$, we have

$$
\varkappa_{6}=\frac{b q\left(984408064224939 q^{2}+20917403154855088 b\right)}{1850072878986734223360} .
$$

Now if we take $\varkappa_{6}=0$, then as $q \neq 0$, either $b=0$ or

$$
b=-\frac{984408064224939}{20917403154855088} q^{2}
$$

If $b=0$, then equations (64) and (65) take the form as below:

$$
\begin{aligned}
& \gamma(\tau)=g\left(\tau^{6}-\frac{1}{7}\right), \\
& \delta(\tau)=q\left(\tau^{6}-\frac{1}{7}\right) .
\end{aligned}
$$

Defining $\sigma(\tau)=\tau^{7}-\tau$, we see $\dot{\sigma}(\tau)=7 \tau^{6}-1$; also, $\sigma(0)=\sigma(1)$. So, it can be written as

$$
\begin{aligned}
& \gamma(\tau)=\frac{g}{7} \dot{\sigma}, \\
& \delta(\tau)=\frac{q}{7} \dot{\sigma} .
\end{aligned}
$$

By Theorem 3, having $f(\sigma)=g / 7$ and $g(\sigma)=q / 7$, the origin is a center. So, we take that $b \neq 0$. By using (70), we have $\varkappa_{7}$ as

$$
\varkappa_{7}=-\frac{132769951 q^{4}\left(-131733796145486983522308400541863 q^{2}+36113870596748585190134519660544 g\right)}{143608906065580564710660947856402888834825584640} .
$$

If $\varkappa_{7}=0$, recalling that $q \neq 0$ (considered above), then Holding (74), we find $g=\frac{131733796145486983522308400541863}{36113870596748585190134519660544} q^{2}$.

$$
\varkappa_{8}=\frac{4236266672814893139248947441614198027669758565038839578999}{348891763171305362071787861985267233073102761553912581534515200} q^{7} .
$$

As $q \neq 0$, we cannot proceed further. We conclude that multiplicity of class $C_{8,6}$ is 8 , i.e., $\mu_{\max }\left(C_{8,6}\right) \geq 8$.
(3) For the class $C_{8,5}$, the degree of $\gamma(\tau)=8$ and $\delta(\tau)=5$; we suppose that $c=d=g=h=l=m=$ 
$n=o=q=r=0$ in system (44). By using Theorem 2 in this case, we calculate

$$
\begin{aligned}
& \varkappa_{2}=k+\frac{p}{6} \\
& \varkappa_{3}=a+\frac{b}{2}+\frac{e}{5}+\frac{f}{6}+\frac{i}{9} .
\end{aligned}
$$

Thus, multiplicity of $\mathscr{Z}=0$ is $x=2$ if $x_{2} \neq 0$. And multiplicity $x=3$ if $x_{2}=0$ but $x_{3} \neq 0$. Suppose $\varkappa_{2}=\varkappa_{3}=0$; then, by using value of $a$ and $j, \delta(\tau)$ and $\gamma(\tau)$ are given as follows:

$$
\gamma(\tau)=b\left(\tau-\frac{1}{2}\right)+e\left(\tau^{4}-\frac{1}{5}\right)+f\left(\tau^{5}-\frac{1}{6}\right)+i\left(\tau^{8}-\frac{1}{9}\right),
$$

$\delta(\tau)=p\left(\tau^{5}-\frac{1}{6}\right)$

We calculate $\varkappa_{4}$ as

$$
\varkappa_{4}=-\frac{p(-176 i+120 e+825 b)}{166320} .
$$

If $\varkappa_{4}=0$, either $p=0$ or

$$
i=\frac{120}{176} e+\frac{825}{176} b
$$

If $p=0$, then $\varkappa_{2}=0$, and $k=0$. As a result, $\delta(\tau)=0$. Also, $\varkappa_{3}=0$ reflects that the mean value $\gamma(\tau)=0$. From
Corollary 1 , origin is the center. Hence, we substitute that $p \neq 0$. If (80) holds, we have

$$
\varkappa_{5}=-\frac{5 p^{2}(-1976 e+42075 b)}{7763019264} .
$$

Now if $\varkappa_{5}=0$, either $p=0$ or $-1976 e+42075 b=0$. But we have already taken $p \neq 0$. So, we take

$$
e=\frac{42075}{1976} b \text {. }
$$

We have $\varkappa_{6}$ as

$$
\varkappa_{6}=\frac{b p\left(93192188 p^{2}+958055593 b\right)}{375931952547840} .
$$

If $x_{6}=0$, then either $p \neq 0, \quad b=0$ or $93192188 p^{2}+958055593 b=0$. If $b=0$, then from (82), $e=o$. By using these values in (80), we see $i=0$. So, equations (77) and (78) can be written as

$$
\begin{aligned}
& \gamma(\tau)=f\left(\tau^{5}-\frac{1}{6}\right), \\
& \delta(\tau)=p\left(\tau^{5}-\frac{1}{6}\right) .
\end{aligned}
$$

Defining $\sigma(\tau)=\tau^{6}-\tau$, we see $\dot{\sigma}(\tau)=6 t^{5}-1$; also, $\sigma(0)=\sigma(1)$. From Theorem 3, the origin is a center along $f(\sigma)=(f / 6)$ and $g(\sigma)=(p / 6)$. We substitute $b \neq 0$. By using (82), we proceed for $\varkappa_{7}$ as

$$
\varkappa_{7}=-\frac{1226213 p^{4}\left(-167427531234665540327 p^{2}+44481776203542888210 f\right)}{1696485821872625651060876535652800} .
$$

If $\varkappa_{7}=0$, recalling that $p \neq 0$ (considered above), then

If (86) holds, we proceed towards $\varkappa_{8}$ as

$$
f=\frac{167427531234665540327}{44481776203542888210} p^{2} .
$$

$$
\varkappa_{8}=\frac{3038511903357314551699258427435859409877}{21491717862570679063691665355228636923726082688000} p^{7}
$$

$\varkappa_{8}$ is a constant multiple of $p^{7}$; as $q \neq 0$, we cannot proceed further. Hence, we conclude that multiplicity of class $C_{8,5}$ is 8 , i.e., $\mu_{\max }\left(C_{8,5}\right) \geq 8$.

Theorem 8. For class $C_{7,7}$ both $\gamma(\tau)$ and $\delta(\tau)$ have degree 7 with the variable $2 \tau$. Then, for equation

$$
\dot{\mathscr{Z}}=\gamma(\tau) \mathscr{Z}^{3}+\delta(\tau) \mathscr{Z}^{2},
$$

$\mu_{\max }\left(C_{7,7}\right) \geq 8$
Proof. Let

$$
\begin{aligned}
& \gamma(\tau)=a+b(2 \tau)+f(2 \tau)^{5}+g(2 \tau)^{6}+h(2 \tau)^{7}, \\
& \delta(\tau)=j+q(2 \tau)^{7} .
\end{aligned}
$$

By Theorem 2, we have

$$
\begin{aligned}
& \varkappa_{2}=16 q+j, \\
& \varkappa_{3}=a+b+\frac{64}{7} g+\frac{16}{3} f+16 h .
\end{aligned}
$$


Thus, multiplicity of $\omega=0$ is $x=2$ if $x_{2} \neq 0$ and $x=3$ if $\varkappa_{2}=0$ but $x_{3} \neq 0$. If $\varkappa_{2}=\varkappa_{3}=0$, from (90) and (91), we substitute $j=-16 q$ and $a=-b-(64 / 7) g-(16 / 3) f-16 h$. Now $\delta(\tau)$ and $\gamma(\tau)$ take the form as

$$
\begin{aligned}
\gamma(\tau)= & b(2 \tau-1)+f\left[(2 \tau)^{5}-\frac{16}{3}\right] \\
& +g\left[(2 \tau)^{6}-\frac{64}{7}\right]+h\left[(2 \tau)^{7}-16\right], \\
\delta(\tau)= & q\left[(2 \tau)^{7}-16\right] .
\end{aligned}
$$

Substituting $j$ and $a$, we calculate $\varkappa_{4}$ as

$$
\varkappa_{4}=-\frac{8 q(336 g+400 f+147 b)}{945} .
$$

If $\varkappa_{4}=0$, then either $q=0$ or

$$
g=-\frac{400}{336} f-\frac{147}{336} b \text {. }
$$

If $q=0$, from (93), $\delta(\tau)=0$ and $\varkappa_{3}=0$ reflect that $\gamma(\tau)$ has mean value zero. Using Corollary 1 , the origin is a center. We suppose $q \neq 0$ and (95) holds; then,

$$
\varkappa_{5}=-\frac{512 q^{2}(-34 f+1323 b)}{580635} .
$$

If $\varkappa_{5}=0$, then as taken above, $q \neq 0$ implies that

$$
f=\frac{1323}{34} b
$$

Now by using (97), we calculate $\varkappa_{6}$ :

$$
\varkappa_{6}=\frac{28 b q\left(18243730176 q^{2}+234430835 b\right)}{41989265175} .
$$

If $\varkappa_{6}=0$, either $b=0$ or

$$
b=-\frac{18243730176}{234430835} q^{2}
$$

as $q \neq 0$. Now if $b=0, f=0$ gives $g=0$. As a result, equations (92) and (97) take the form:

$$
\begin{aligned}
& \gamma(\tau)=h\left[(2 \tau)^{7}-16\right] \\
& \delta(\tau)=p\left[(2 \tau)^{7}-16\right] .
\end{aligned}
$$

Consider $\sigma(\tau)=(2 \tau)^{8}-256 t$; then, $\dot{\sigma}(\tau)=16\left[(2 \tau)^{7}-\right.$ 16] ; also, $\sigma(0)=\sigma(1)$. We write $\gamma(\tau)$ and $\delta(\tau)$ as

$$
\begin{aligned}
& \gamma(\tau)=16 h \dot{\sigma}, \\
& \delta(\tau)=16 p \dot{\sigma} .
\end{aligned}
$$

By Theorem 3, origin is a center having $f(\sigma)=16 h$ and $g(\sigma)=16 p$. Therefore, we conclude that $b \neq 0$. By taking, $\varkappa_{7}$ is computed as

$$
\varkappa_{7}=-\frac{8543709116235776 q^{4}\left(35792018807075552 q^{2}+32995285488045 h\right)}{223319838020134861120561875} .
$$

If $\varkappa_{7}=0$, recalling that $q \neq 0$ (taken above), we have

If (103) holds, we calculate

$$
h=-\frac{35792018807075552}{32995285488045} q^{2}
$$

$$
\varkappa_{8}=\frac{107535112433233332507776575245060923092413448192}{1713318730986483405512695232955553868915625} q^{7} \text {, }
$$

which is a constant multiple of $q^{7}$ and also nonzero as $q \neq 0$. Hence, we conclude that the multiplicity is 8 , i.e., $\mu_{\max }\left(C_{7,7}\right) \geq 8$.

\section{Theorem 9. For equation}

$$
\dot{\mathscr{Z}}=\gamma(\tau) \mathscr{Z}^{3}+\delta(\tau) \mathscr{Z}^{2}+\varkappa_{1} \mathscr{Z}+\varkappa_{2},
$$

consider

$$
\begin{aligned}
& \gamma(\tau)=-v_{1}+\varepsilon_{5}+b(2 \tau)+f(2 \tau)^{5}+g(2 \tau)^{6}+h(2 \tau)^{7}, \\
& \delta(\tau)=-16 q+\varepsilon_{6}+128 q t^{7} .
\end{aligned}
$$

Here,

$$
\begin{aligned}
& v_{1}-b-\frac{64}{7} g-\frac{16}{3} f-16 h, \\
& b=-\frac{18243730176}{234430835} q^{2}+\varepsilon_{2}, \\
& g=\frac{630548924208}{173274965} q^{2}-\frac{12719}{272} \varepsilon_{2}-\frac{25}{21} \varepsilon_{3}+\varepsilon_{4}, \\
& f=-\frac{12068227511424}{3985324195} q^{2}+\frac{1323}{34} \varepsilon_{2}+\varepsilon_{3}, \\
& h=-\frac{35792018807075552}{32995285488045} q^{2}+\varepsilon_{1} .
\end{aligned}
$$

If $\varepsilon_{p}(1 \leq p \leq 6), \varkappa_{1}$ and $\varkappa_{2}$ are chosen to be nonzero and

$$
\left|\varkappa_{2}\right| \ll\left|\varkappa_{1}\right| \ll\left|\varepsilon_{6}\right| \ll\left|\varepsilon_{5}\right| \ll \ldots \ll\left|\varepsilon_{1}\right| \text {. }
$$


Then, (105) has eight distinct nontrivial real periodic solutions.

Proof. If we substitute $\varepsilon_{p}$ for $(1 \leq p \leq 6)$, the proof is similar to Theorem 6 . Hence, it is omitted.

\section{Examples}

In this section, we are presenting some examples which are helpful to show the applicability of the methods presented.

Example 1. Consider the differential equation:

$$
\frac{\mathrm{d} z}{\mathrm{~d} t}=\left(e^{t}\right) z^{3}+(\sin t) z^{2}
$$

Here $\gamma(t), \delta(t)$ are transcendental functions, but we use the power series representation by neglecting the terms " $t$ " " for $n>5$, so we have $\gamma(t)=e^{t}=a+b t+c\left(t^{2} / 2 !\right)+d\left(t^{3} / 3 !\right)+$ $e\left(t^{4} / 4 !\right)+f\left(t^{5} / 5 !\right)$ and $\delta(t)=\sin t=h t-i\left(t^{3} / 3 !\right)+j\left(t^{5} / 5 !\right)$. Here $a, b, c, d, e, f, h, i, j$ are constants having same value equal to 1 . The solution of the above equation is given below.

Solution. We substitute $i=e=0$, for suitable restriction of the coefficients. By using Theorem 2, we calculate

$$
\begin{aligned}
& \eta_{2}=\frac{1}{2} h+\frac{1}{720} j, \\
& \eta_{3}=a+\frac{1}{2} b+\frac{1}{6} c+\frac{1}{24} d+\frac{1}{720} f .
\end{aligned}
$$

Thus, multiplicity of $z=0$ is $\mu=2$ if $\eta_{2} \neq 0$. And multiplicity $\mu=3$ if $\eta_{2}=0$ but $\eta_{3} \neq 0$. If $\eta_{2}=\eta_{3}=0$, then we take $h=-2 / 720 j$, and

$$
a=-\frac{1}{2} b-\frac{1}{6} c-\frac{1}{24} d-\frac{1}{720} f .
$$

By using these values, $\eta_{4}$ is calculated as

$$
\eta_{4}=-\frac{j(5 f+192 d+768 c+1800 b)}{43545600} .
$$

If $\eta_{4}=0$, then either $j=0$ or

$$
f=-\frac{192}{5} d-\frac{768}{5} c-\frac{1800}{5} b \text {. }
$$

If $j=0$, then $h=0$; this shows that $\delta(t)=0$, and $\eta_{3}=0$ reflects that mean value of $\gamma(t)$ is zero. By Corollary 1 , the origin is a center. Suppose $j \neq 0$; if (113) holds, we calculate $\eta_{5}$ as

$$
\eta_{5}=\frac{j^{2}(5071 d+16384 c)}{56043187200000} .
$$

If $\eta_{5}=0$, then $j \neq 0$ (taken above); we substitute $d=-(16384 / 5071) c$ and calculate $\eta_{6}$ as

$$
\eta_{6}=\frac{c j\left(56201893 j^{2}+115870113000 c\right)}{13964771180634962688000} .
$$

If $\eta_{6}=0$, then either $c=0$ or

$$
c=-\frac{56201893}{115870113000} j^{2}
$$

because $j \neq 0$ (already taken above). If $c=0$, then $d=0$; substituting these values in (113) gives $f=-(1800 / 5) b$, and (111) results in $a=0$. By using these values, $\gamma(t)$ and $\delta(t)$ take the following form:

$$
\begin{aligned}
& \gamma(t)=f\left(\frac{t^{5}}{5 !}-\frac{2 t}{720}\right), \\
& \delta(t)=j\left(\frac{t^{5}}{5 !}-\frac{2 t}{720}\right) .
\end{aligned}
$$

Let $\sigma(t)=\left(t^{6} / 5 !\right)-\left(t^{2} / 720\right)$; then, $\quad \dot{\sigma}(t)=\left(6 t^{5} / 5 !\right)$ $(2 t / 720)$. Also, $\sigma(0)=\sigma(1)$. So, we can write $\gamma(t)=(1 / 6 !) f \dot{\sigma}$ and $\delta(t)=(1 / 6 !) j \dot{\sigma}$. From Theorem 3, origin is a center having $f(\sigma)=(1 / 6 !) f$ and $g(\sigma)=(1 / 6 !) j$. Suppose that $c \neq 0$; by using (116), we have $\eta_{7}$ as follows:

$$
\eta_{7}=-\frac{11083 j^{4}\left(-125099092877941 j^{2}+1849231385825760000 b\right)}{21873119144875211817269842176000000000} .
$$

Recalling that $j \neq 0$ (considered above), if $\eta_{7}=0$, then we and calculate $\eta_{8}$ as put

$$
b=\frac{125099092877941}{1849231385825760000} j^{2},
$$

$$
\eta_{8}=\frac{83017743756733094728041979679}{218732377679448920984181770497759873277952000000000000} j^{7},
$$


which is a constant multiple of $j^{7}$, and $j \neq 0$. Thus, we conclude that (109) has eight periodic solutions.

Example 2. Consider the differential equation:

$$
\frac{\mathrm{d} z}{\mathrm{~d} t}=\gamma(t) z^{3}+\delta(t) z^{2}
$$

with $\gamma(t)=$ equation of circle $=a x^{2}+b y^{2}+c x+d y+e$ and $\delta(t)=$ point circle $=$ circle with $($ center $(0,0)$ and radius $=0)=g x^{2}+h y^{2}$; we then calculate the periodic solutions as given below.

Solution. By using Theorem 2, we calculate

$$
\begin{aligned}
& \eta_{2}=\frac{1}{3} g+\frac{1}{3} h, \\
& \eta_{3}=\frac{1}{3} a+\frac{1}{3} b+\frac{1}{2} c+\frac{1}{2} d+e .
\end{aligned}
$$

Thus, multiplicity of $z=0$ is $\mu=2$ if $\eta_{2} \neq 0$. And multiplicity $\mu=3$ if $\eta_{2}=0$ but $\eta_{3} \neq 0$. If $\eta_{2}=\eta_{3}=0$, then we put $g=-h$, and $e=-(1 / 3) a-(1 / 3) b-(1 / 2) c-(1 / 2) d$. By using these values, we get

$$
\eta_{4}=-\frac{h(-5 b-4 d+5 a+4 c)}{720} .
$$

If $\eta_{4}=0$, then either

$$
b=-\frac{4}{5} d+\frac{5}{5} a+\frac{4}{5} c
$$

or $h=0$. We get $\delta(t)=0$ for $h=0$, and $\eta_{3}=0$ shows that mean value of $\gamma(t)$ is zero. So, by Corollary 1 , the origin is a center. Suppose that $h \neq 0$. Now by using (124), we calculate $\eta_{5}=0$ as

$$
\eta_{5}=\frac{h^{2}(73 d+1280 a+1097 c)}{1701000} .
$$

If $\eta_{5}=0$, recalling that $h \neq 0$, we put

$$
d=-\frac{1280}{73} a-\frac{1097}{73} c
$$

and we calculate

$$
\eta_{6}=\frac{h(128 a+117 c)\left(163845 a+131076 c+1825 h^{2}\right)}{3988436760} .
$$

If $\eta_{6}=0$, then either $a=-(117 / 128) c$ or

$$
a=-\frac{131076}{163845} c-\frac{1825}{163845} h^{2},
$$

because $h \neq 0$. If $a=-(117 / 128) c$ holds but (128) does not hold, we calculate $\eta_{7}$ as

$$
\eta_{7}=\frac{c h^{2}\left(-12845056 h^{2}+24891165 c\right)}{100429391462400} .
$$

Now, if (128) holds but $a=-(117 / 128) c$ does not hold, we calculate $\eta_{7}$ as

$$
\eta_{7}=-\frac{h^{2}\left(-1248682406400 c h^{2}+6393449027394 c^{2}+97998898375 h^{4}\right)}{3656745734764122000} .
$$

From (130), we cannot substitute any value to proceed further, and if $\eta_{7}=0$ for equation (129), we substitute $c=$ $(12845056 / 24891165) h^{2}$ and calculate $\eta_{8}=0$. Hence, in both the cases, we succeeded to calculate $\eta_{7}$ as nonzero. Thus, we conclude that (121) has seven periodic solutions.

\section{Conclusion and Discussion}

In this paper, we presented the maximum number of periodic orbits which are usually called limit cycles. Limit cycles are closed paths that are isolated from set of all periodic orbits. To find the maximum number of limit cycles, the perturbation technique has been adopted. We adopt a systematic procedure to define coefficients of higher-order polynomials. We furnished the maximum number of periodic solutions for some classes $C_{7,7}, C_{8,5}, C_{8,6}, C_{8,7}$. They are found with 8 periodic solutions at most. To find out more periodic solutions, keeping the motivation of second part of Hilbert's sixteenth problem in our mind, we developed formulas for $\xi_{10}$ and $\varkappa_{10}$. With the help of newly developed formulas, we are able to calculate multiplicity 10 for class $C_{9,2}$. This multiplicity is the highest known multiplicity in the literature to date. We also verified the results by carrying out some examples given in Section 5, which shows that presented methods are new, applicable, and authentic.

\section{Data Availability}

The data used to support the findings of this study are available from the corresponding author upon request.

\section{Conflicts of Interest}

The authors declare that they have no conflicts of interest.

\section{Authors' Contributions}

Saima Akram carried out the proof of Theorem 5, Theorem 6, and Corollary 2 and drafted the manuscript. Allah Nawaz carried out the proof of Theorem 7. Humaira Kalsoom carried out the proof of Theorem 8. Muhammad Idrees provided Example 1. Yu-Ming Chu provided the main idea and Example 2, completed the final revision, and submitted the article. All authors read and approved the final manuscript. 


\section{Acknowledgments}

This study was supported by the National Natural Science Foundation of China (grant no. 61673169).

\section{References}

[1] G. Faye, An Introduction to Bifurcation Theory, NeuroMathComp Laboratory, INRIA, CNRS, ENS, Paris, France, 2011.

[2] A. L. Neto, "On the number of solutions of the equation $\mathrm{d} x / \mathrm{d} t=\sum_{j=0}^{n} a_{j}(t) t^{\prime}, \quad 0 \leq t \leq 1 \quad$ for which $x(0)=x(1), "$ Inventiones Mathematicae, vol. 59, no. 1, pp. 67-76, 1980.

[3] S. Kadry, G. Alferov, and A. Sharlay, "Almost periodic solutions of first order ordinary differential equations," Mathematics, vol. 6, p. 171, 2018.

[4] M. A. M. Alwash and N. G. Llyod, "Non-autonomous equation related to polynomial two-dimensional system," Proceedings of royal society Edinburgh, vol. 105, pp. 129-152, 1987.

[5] N. G. Llyod, "The number of periodic solutions of the equation $\quad Z=z^{n}+p_{1}(t) z^{n-1}+P_{2}(t) z^{n-2}+\cdots+P_{n}(t)$." Proceedings of London Mathematical Society, vol. 27, no. 3, pp. 667-700, 1973.

[6] A. Nawaz, "Bifurcation of periodic solutions of certain classes of cubic non-autonomous differential equations," M.Phil thesis, Hec, Islamabad, Pakistan, pp. 1-104, 2018.

[7] D. Hilbert, "Mathematical problems," Bulletin of the American Mathematical Society, vol. 8, no. 10, pp. 437-480, 1902.

[8] N. G. Llyod, "Limit cycles of certain polynomial systems, nonlinear functional analysis and its applications," in Natoo Asi Series C, S. P. Singh, Ed., vol. 173, pp. 317-326, 1986.

[9] S. Akram, A. Nawaz, N. Yasmin, A. Ghaffar, D. Baleanu, and K. S. Nisar, "Periodic solutions of some classes of one dimensional non-autonomous system," Frontiers in Physics, 2020.

[10] N. Yasmin, "Bifurcating periodic solutions of polynomial system," Punjab university Journal of Mathematics, vol. 34, pp. 43-46, 2001.

[11] A. B. Makar, K. E McMartin, M Palese, and T. R Tephly, "Formate assay in body fluids: application in methanol poisoning," Biochemical Medicine, vol. 13, pp. 117-126, 1975.

[12] M. A. M. Alwash, "Periodic solutions of polynomial nonautonomous differential equations," Electronic Journal of Differential Equations, vol. 84, pp. 1-8, 2005. 